\title{
UBIQUITY OF ENTROPIES OF INTERMEDIATE FACTORS
}

\author{
KEVIN MCGOFF AND RONNIE PAVLOV
}

\begin{abstract}
We consider topological dynamical systems $(X, T)$, where $X$ is a compact metrizable space and $T$ denotes an action of a countable amenable group $G$ on $X$ by homeomorphisms. For two such systems $(X, T)$ and $(Y, S)$ and a factor map $\pi: X \rightarrow Y$, an intermediate factor is a topological dynamical system $(Z, R)$ for which $\pi$ can be written as a composition of factor maps $\psi: X \rightarrow Z$ and $\varphi: Z \rightarrow Y$. In this paper we show that for any countable amenable group $G$, for any $G$-subshifts $(X, T)$ and $(Y, S)$, and for any factor map $\pi: X \rightarrow Y$, the set of entropies of intermediate subshift factors is dense in the interval $[h(Y, S), h(X, T)]$. As a corollary, we also prove that if $(X, T)$ and $(Y, S)$ are zerodimensional $G$-systems, then the set of entropies of intermediate zero-dimensional factors is equal to the interval $[h(Y, S), h(X, T)]$. Our proofs rely on a generalized Marker Lemma that may be of independent interest.
\end{abstract}

\section{INTRODUCTION}

In this work, we continue a line of research, initiated by Shub and Weiss [13], concerning the following seemingly basic question: given a topological dynamical system, what can be said about the topological entropies of its factors? For the purposes of this paper, we consider a topological dynamical system to be a pair $(X, T)$, where $X$ is a compact metrizable space and $T$ is an action of a countable amenable group $G$ on $X$ by homeomorphisms. Additionally, a factor of such a system $(X, T)$ is another system $(Y, S)$ for which there exists a continuous surjection $\pi: X \rightarrow Y$ that commutes with the actions of $S$ and $T$. Some general results about the entropies of factors are available [9, 10, 13, but the question above has not been completely resolved. For instance, it is

2010 Mathematics Subject Classification. Primary: 37B10; Secondary: 37B50, 37B40.

Key words and phrases. Symbolic dynamics, amenable, topological entropy.

The first author gratefully acknowledges support from National Science Foundation grants DMS-1613261 and DMS-1847144. The second author gratefully acknowledges the support of a Simons Foundation Collaboration Grant. 
still not known whether every system with positive, finite entropy must have a nontrivial factor with strictly smaller entropy.

Under certain hypotheses, there are some existing results about the entropies of factors. For instance, Lindenstrauss proved [9] that if $(X, T)$ is a finite-dimensional $\mathbb{Z}$-system, then the set of entropies of factors of $(X, T)$ is the entire interval $[0, h(X, T)]$. Furthermore, Lindenstrauss provided examples showing that this is not necessarily the case if $X$ has infinite dimension.

Lindenstrauss also proved what we call a 'relative version' of his result, which concerns the entropies of intermediate factors. Given a countable amenable group $G, G$-systems $(X, T)$ and $(Y, S)$, and a factor map $\pi: X \rightarrow Y$, an intermediate factor is a $G$-system $(Z, R)$ for which $\pi$ can be written as a composition of factor maps $\psi: X \rightarrow Z$ and $\varphi: Z \rightarrow Y$. With this definition, Lindenstrauss showed that if $\pi$ is a factor map from a finite dimensional $\mathbb{Z}$-system $(X, T)$ onto $(Y, S)$, then the set of entropies of intermediate factors is the entire interval $[h(Y, S), h(X, T)][9]$. (The relative version is of course more general than the original result concerning entropies of factors of a single system, since the original result may be obtained by taking $(Y, S)$ to be the trivial factor in the relative version.) Using the notion of mean dimension [11, Lindenstrauss also generalized these results to extensions of minimal $\mathbb{Z}$-systems with zero mean dimension [10].

Here we examine the question in the setting of subshifts, or symbolic dynamical systems. Of course subshifts are zero-dimensional, and therefore previous results about zero-dimensional systems can be applied to subshifts. However, we are interested in the finer question of what can be said about the entropies of intermediate factors, where the intermediate systems must come from a restricted class (such as subshifts or zero-dimensional systems). In this direction, for $\mathbb{Z}$-subshifts, it was shown in [13] that any system with positive entropy must have nontrivial subshift factors of strictly smaller entropy (and in fact that they can be taken arbitrarily close to $h(X, T)$ ). In addition, in [4], in the case where $(X, T)$ is a sofic $\mathbb{Z}$-subshift, it was shown that the set of entropies of subshift factors is dense in the interval $[0, h(X, T)]$, which is in some sense the most that can be hoped for, since a subshift has only countably many subshift factors by the Curtis-Lyndon-Hedlund theorem. A corresponding relative result was also established when $(X, T)$ and $(Y, S)$ are both sofic $\mathbb{Z}$-subshifts.

In this work, we show that the previously mentioned result of [4] holds even when $(X, T)$ is an arbitrary (not necessarily sofic) subshift. Furthermore, we generalize these results from $\mathbb{Z}$-subshifts to $G$-subshifts, where $G$ is an arbitrary countable amenable group. Note 
that the computation/realization of entropies is much more difficult in the setting of general countable amenable groups. For instance, realization of arbitrary entropies for $G$-subshifts has only recently been addressed for general classes of countable amenable groups [3, 14]. In order to establish our main results at this level of generality, we first prove a generalized Marker Lemma for countable groups.

Let us now state our main results. In the following two results, $G$ denotes any countable amenable group, $(X, T)$ and $(Y, T)$ represent $G$-subshifts, $\mathcal{H}_{\text {sub }}(X)$ denotes the set of numbers $r \in \mathbb{R}$ such that there exists a $G$-subshift $(Z, R)$ so that $(X, T)$ factors onto $(Z, R)$ and $h(Z, R)=r$, and $\mathcal{H}_{\text {sub }}^{\pi}(X, Y)$ denotes the set of numbers $r \in \mathbb{R}$ such that there exists a $G$-subshift $(Z, R)$ with $h(Z, R)=r$ and factor maps $\varphi: X \rightarrow Z$ and $\psi: Z \rightarrow Y$ such that $\pi=\psi \circ \varphi$.

Theorem 1.1. Let $G$ be a countable amenable group, and let $(X, T)$ be a $G$-subshift. Then $\mathcal{H}_{\text {sub }}(X)$ is dense in the interval $[0, h(X, T)]$.

The main technique in the proof of Theorem 1.1 is a general version of the classical Krieger Marker Lemma (see [2, [6]), which may be of independent interest; see Section 3. We also establish the following relative version.

Theorem 1.2. Let $G$ be a countable amenable group. Let $(X, T)$ and $(Y, S)$ be $G$-subshifts with a factor map $\pi: X \rightarrow Y$. Then $\mathcal{H}_{\text {sub }}^{\pi}(X, Y)$ is dense in the interval $[h(Y, S), h(X, T)]$.

From these results, we are actually able to establish a similar result about general zero-dimensional systems as well.

In the following result, for zero-dimensional $G$-systems $(X, T)$ and $(Y, S)$ and a factor map $\pi: X \rightarrow Y$, we let $\mathcal{H}_{0}^{\pi}(X, Y)$ denote the set of numbers $r \in \mathbb{R}$ such that there exists a zero-dimensional $G$-system $(Z, R)$ with $h(Z, R)=r$ and factor maps $\varphi: X \rightarrow Z$ and $\psi: Z \rightarrow Y$ such that $\pi=\psi \circ \varphi$.

Theorem 1.3. Let $G$ be a countable amenable group, and let $\pi$ : $(X, T) \rightarrow(Y, S)$ be a factor map between zero-dimensional $G$-systems. Then $\mathcal{H}_{0}^{\pi}(X, Y)=[h(Y, S), h(X, T)]$.

We note that this result provides more information about the aforementioned result of [9] when $(X, T)$ and $(Y, S)$ are zero-dimensional: in this case, not only do we know that every entropy in $[h(Y, S), h(X, T)]$ can be achieved by factors, but we now know that those factors can always be chosen to be zero-dimensional.

The rest of the paper is organized as follows. Section 2 contains necessary background and notation, Sections 3 and 4 contain some 
preliminary results required for later proofs, and Sections 5, 6, and 7 contain the proofs of Theorems $1.1,1.3$.

\section{ACKNOWLEDGMENTS}

The authors would like to thank Uijin Jung for introducing these questions to them and for many useful conversations. Additionally, the authors wish to thank Mike Boyle for asking them about zerodimensional factors, thus motivating Theorem 1.3. The authors also wish to thank Tomasz Downarowicz for helpful conversations regarding zero-dimensional factors and topological joinings.

\section{BACKGROUND AND NOTATION}

2.1. Countable amenable groups. Let $G$ be a countable amenable group. For sets $A, K \subset G$, we let $A K=\{a k: a \in A, k \in K\}$ and $A \Delta K=(A \backslash K) \cup(K \backslash A)$. A sequence $\left\{F_{n}\right\}_{n=1}^{\infty}$ of finite subsets of $G$ is called a $F \varnothing l n e r$ sequence if for each finite set $K \subset G$, we have

$$
\lim _{n} \frac{\left|K F_{n} \Delta F_{n}\right|}{\left|F_{n}\right|}=0
$$

The existence of a Følner sequence is equivalent to the amenability of the group $G$. Let $\left\{F_{n}\right\}_{n=1}^{\infty}$ be a Følner sequence, which we fix here and use throughout the paper.

Definition 2.1. Suppose $U$ and $K$ are non-empty finite subsets of $G$ and $\delta>0$. We say that $U$ is $(K, \delta)$-invariant if

$$
\frac{|K U \triangle U|}{|U|}<\delta \text {. }
$$

Note that the definition of Følner sequence yields that for any finite set $K \subset G$ and any $\delta>0$, the set $F_{n}$ is $(K, \delta)$-invariant for all large enough $n$.

Definition 2.2. Suppose $\left\{A_{1}, \ldots, A_{r}\right\}$ is a collection of finite sets of $G$. We say that the collection is $\delta$-disjoint if there exist subsets $\left\{B_{1}, \ldots, B_{r}\right\}$ such that

(1) $B_{i} \subset A_{i}$ for all $i$,

(2) for $i \neq j$, we have $B_{i} \cap B_{j}=\varnothing$, and

(3) $\left|B_{i}\right| /\left|A_{i}\right|>1-\delta$ for all $i$.

Definition 2.3. Suppose $\left\{A_{1}, \ldots, A_{r}\right\}$ is a collection of finite sets of $G$, and let $B \subset G$. We say that the collection $\alpha$-covers $B$ if

$$
\left|B \cap\left(\bigcup_{i} A_{i}\right)\right| \geq \alpha|B|
$$


Definition 2.4. ([12]) For $\delta>0$, a collection of finite sets $\left\{T_{1}, \ldots, T_{N}\right\}$ is said to $\delta$-quasitile a group $G$ (or to be a set of $\delta$-quasitiles for $G$ ) if $\{e\} \in T_{1} \subset \cdots \subset T_{N}$ and for any finite set $D \subset G$, there are finite sets $C_{i} \subset G$, for $1 \leq i \leq N$, such that

(1) for fixed $i$, the collection $\left\{T_{i} c: c \in C_{i}\right\}$ is $\delta$-disjoint

(2) for $i \neq j, T_{i} C_{i} \cap T_{j} C_{j}=\varnothing$, and

(3) the collection $\left\{T_{1} C_{1}, \ldots, T_{N} C_{N}\right\}(1-\delta)$-covers $D$.

The fundamental result of Ornstein and Weiss [12, Theorem 6] states that for any $\delta>0$, there exists $N$ such that for any finite set $K$ and any $\delta^{\prime}>0$, there exist finite sets $\left\{T_{1}, \ldots, T_{N}\right\}$ that are $\left(K, \delta^{\prime}\right)$-invariant and that $\delta$-quasi-tile $G$. We remark that by making $\delta^{\prime}>0$ small enough and $K$ large enough, one may force $\left|T_{1}\right|=\min _{i}\left|T_{i}\right|$ to be arbitrarily large.

For a collection of quasitiles $\left\{T_{1}, \ldots, T_{N}\right\}$, we refer to any collection $\left\{C_{1}, \ldots, C_{N}\right\}$ satisfying (1)-(3) above as a collection of center sets corresponding to $D$. If $\left\{C_{1}, \ldots, C_{N}\right\}$ is a collection of center sets for $D$, then let $C_{i}^{\prime}=\left\{c \in C_{i}: T_{i} c \cap D \neq \varnothing\right\}$, and observe that the collection $\left\{C_{1}^{\prime}, \ldots, C_{N}^{\prime}\right\}$ is again a collection of center sets for $D$. Thus, for a collection of quasitiles $\left\{T_{1}, \ldots, T_{N}\right\}$ and finite $D \subset G$, there exists a collection of center sets $\left\{C_{1}, \ldots, C_{N}\right\}$, and we may assume without loss of generality that for all $c \in C_{i}$, we have $T_{i} c \cap D \neq \varnothing$.

Lemma 2.5. Let $\delta \in(0,1)$. Suppose that $\left\{S_{1}, \ldots, S_{N}\right\}$ is a collection of finite sets that $\delta$-quasitiles $G$. Let $m=\left|S_{1}\right|=\min \left\{\left|S_{1}\right|, \ldots,\left|S_{N}\right|\right\}$, and $S=\cup_{i} S_{i}=S_{N}$. Then for all large enough $n$, there exists $C=$ $C(n) \subset G$ such that $\{S c: c \in C\}(1-\delta)$-covers $F_{n}$ and

$$
|C| \leq \frac{(1+\delta)\left|F_{n}\right|}{(1-\delta) m}
$$

Proof. Since $\left\{F_{n}\right\}_{n=1}^{\infty}$ is a Følner sequence, for all large enough $n$, we have

$$
\frac{\left|S S^{-1} F_{n} \triangle F_{n}\right|}{\left|F_{n}\right|}<\delta .
$$

Fix $n$ satisfying this condition. Since $\left\{S_{1}, \ldots, S_{N}\right\}$ is a collection of $\delta$-quasitiles, there exists a collection of center sets corresponding to the set $F_{n}$ with the additional property that for each $c \in C_{i}$, we have $S_{i} c \cap F_{n} \neq \varnothing$.

Let $C=\bigcup_{i} C_{i}$. Then

$$
A=\bigcup_{i} S_{i} C_{i} \subset S C,
$$


and since $A(1-\delta)$-covers $F_{n}$, we see that $S C(1-\delta)$-covers $F_{n}$.

Now let us estimate $|C|$. Let $C_{i}=\left\{c_{1}^{i}, \ldots, c_{M_{i}}^{i}\right\}$, and let $A_{k}^{i}=S_{i} c_{k}^{i}$, for $k=1, \ldots, M_{i}$. Then the collection $\left\{A_{k}^{i}: 1 \leq i \leq N, 1 \leq k \leq M_{i}\right\}$ is $\delta$-disjoint (by the quasitile properties (1) and (2)). Then there exist sets $B_{k}^{i} \subset A_{k}^{i}$ such that $\left|B_{k}^{i}\right| \geq\left|A_{k}^{i}\right|(1-\delta)$ and if $B_{k}^{i} \cap B_{\ell}^{j} \neq \varnothing$, then $i=j$ and $k=\ell$. Then we have

$$
\begin{aligned}
\bigcup_{i=1}^{N} \bigcup_{k=1}^{M_{i}} B_{k}^{i} \mid & =\sum_{i=1}^{N} \sum_{k=1}^{M_{i}}\left|B_{k}^{i}\right| \\
& \geq \sum_{i=1}^{N} \sum_{k=1}^{M_{i}}(1-\delta)\left|A_{k}^{i}\right| \\
& =(1-\delta) \sum_{i=1}^{N} \sum_{k=1}^{M_{i}}\left|S_{i}\right| \\
& =(1-\delta) \sum_{i=1}^{N}\left|S_{i}\right| \cdot\left|C_{i}\right| \\
& \geq(1-\delta) m \sum_{i=1}^{N}\left|C_{i}\right| \\
& \geq(1-\delta) m|C| .
\end{aligned}
$$

Fix $1 \leq i \leq N$ and $1 \leq k \leq M_{i}$. Recall that $A_{k}^{i} \cap F_{n}=S_{i} c_{k}^{i} \cap F_{n} \neq \varnothing$. Let $g \in S_{i} c_{k}^{i} \cap F_{n}$. Then there exists $s \in S_{i}$ such that $g=s c_{k}^{i}$, which implies that $c_{k}^{i}=s^{-1} g \in S^{-1} F_{n}$, and hence $S c_{k}^{i} \subset S S^{-1} F_{n}$. Then we see that

$$
\begin{aligned}
\left|\bigcup_{i=1}^{N} \bigcup_{k=1}^{M_{i}} B_{k}^{i}\right| & \leq\left|\bigcup_{i=1}^{N} \bigcup_{k=1}^{M_{i}} A_{k}^{i}\right| \\
& \leq\left|S S^{-1} F_{n}\right| \\
& \leq(1+\delta)\left|F_{n}\right|,
\end{aligned}
$$

where we have used (2.1). Combining the above inequalities gives

$$
(1-\delta) m|C| \leq\left|\bigcup_{i=1}^{N} \bigcup_{k=1}^{M_{i}} B_{k}^{i}\right| \leq(1+\delta)\left|F_{n}\right|,
$$

from which we conclude that

$$
|C| \leq \frac{(1+\delta)\left|F_{n}\right|}{(1-\delta) m}
$$

as desired. 
2.2. Topological and symbolic dynamics. We present some basic definitions from topological and symbolic dynamics; for a more thorough introduction to symbolic dynamics, see [8].

Definition 2.6. Let $G$ be a countable amenable group. A G-system is a pair $(X, T)$, where $X$ is a compact metrizable space and $T=\left(T^{g}\right)_{g \in G}$ is an action of $G$ on $X$ by homeomorphisms.

Definition 2.7. A factor map from a $G$-system $(X, T)$ to a $G$-system $(Y, S)$ is a surjective function $\varphi: X \rightarrow Y$ that is continuous and commutes with the actions of $S$ and $T$. A conjugacy is a factor map that is also injective. When $\varphi: X \rightarrow Y$ is a factor map, we refer to $(Y, S)$ as a factor of $(X, T)$.

Definition 2.8. For any finite alphabet $\mathcal{A}$ and countable amenable group $G$, the full $G$-shift over $\mathcal{A}$ is the set $\mathcal{A}^{G}$, which is viewed as a compact topological space with the (discrete) product topology.

Definition 2.9. A pattern over $\mathcal{A}$ is any function $w$ from a finite set $S \subset G$ to $\mathcal{A}$, in which case $S$ is called the shape of $w$.

Definition 2.10. The shift action $\sigma=\left(\sigma^{g}\right)_{g \in G}$ is the action of $G$ on $\mathcal{A}^{G}$ by automorphisms defined by $\left(\sigma^{g}(x)\right)(h)=x(h g)$ for all $g, h \in G$.

Definition 2.11. A $G$-subshift is a closed subset of a full shift $\mathcal{A}^{G}$ that is invariant under $\sigma$.

Any subshift $X$ is a compact space with the induced topology from $\mathcal{A}^{G}$, and so $\left(X,\left.\sigma\right|_{X}\right)$ is a topological $G$-dynamical system. In fact, this topology on $X$ is also generated by the ultra-metric given by

$$
d(x, y)=2^{-\min \left\{n: x_{g_{n}} \neq y_{g_{n}}\right\}},
$$

where $G=\left\{g_{n}\right\}_{n=0}^{\infty}$ is an enumeration of $G$.

Definition 2.12. For any $G$-subshift $X$ and finite $S \subset G$, the $S$ language of $X$, denoted by $\mathcal{L}_{S}(X)$, is the set of all patterns $w$ with shape $S$ which appear as subpatterns of points of $X$, i.e., for which there exists $x \in X$ and $g \in G$ with $x(S g)=w$.

Definition 2.13. For any $G$-subshift $X$ and $w \in \mathcal{L}_{S}(X)$, the cylinder set $[w]$ is the set of all $x \in X$ with $x(S)=w$.

All factor maps between subshifts have a simple combinatorial description.

Definition 2.14. Given alphabets $\mathcal{A}$ and $\mathcal{B}$, a finite set $S \subset G$, and a function $f: \mathcal{A}^{S} \rightarrow \mathcal{B}$, the sliding block code induced by $S$ and $f$ is the $\operatorname{map} \varphi: \mathcal{A}^{G} \rightarrow \mathcal{B}^{G}$ defined by

$$
(\varphi(x))(g)=f(x(S g))
$$


for all $x \in \mathcal{A}^{G}$ and $g \in G$. A 1-block code is a sliding block code when $S=\{e\}$.

If $X$ and $Y$ are subshifts, then for every factor map $\pi: X \rightarrow Y$, there is a sliding block code $\varphi$ such that $\pi=\left.\varphi\right|_{X}$; this is the classical CurtisLyndon-Hedlund theorem when $G=\mathbb{Z}$, and the proof is essentially the same for general $G$. Even more is true: up to conjugacy, every factor map can be written as a 1-block code, i.e., if $\varphi: X \rightarrow Y$ is a factor map, then there exists a conjugacy $\psi$ on $X$ and a 1-block code $\rho$ on $\psi(X)$ so that $\varphi=\rho \circ \psi$.

2.3. Entropy. When $G$ is a countable amenable group, one may associate to each $G$-system $(X, T)$ an extended real number called the topological entropy of $(X, T)$. The entropy of a system quantifies its complexity and serves as an important conjugacy invariant in the study of topological dynamical systems. The entropy theory for countable amenable group actions is well-established [5, 12]. Here we state the main entropy-related definitions and properties that we require elsewhere in the paper. We begin with topological entropy and topological conditional entropy.

Definition 2.15. Suppose $\pi: X \rightarrow Y$ is a factor map between $G$ systems $(X, T)$ and $(Y, S)$. Let $\mathcal{U}$ be a finite open cover of $X$. For any subset $K \subset X$, let $N(\mathcal{U} \mid K)$ be the minimal cardinality of any subcover of $\mathcal{U}$ that covers $K$. Define

$$
h(T, \mathcal{U} \mid S)=\lim _{n} \frac{1}{\left|F_{n}\right|} \log \sup _{y \in Y} N\left(\mathcal{U}_{F_{n}} \mid \pi^{-1}(y)\right),
$$

where $\mathcal{U}_{F_{n}}=\bigvee_{g \in F_{n}} T^{g^{-1}} \mathcal{U}$ and $\left\{F_{n}\right\}$ is a Følner sequence. Then the conditional entropy of $(X, T)$ with respect to $(Y, S)$ is given by

$$
h(T \mid S)=\sup _{\mathcal{U}} h(T, \mathcal{U} \mid S),
$$

where the supremum is taken over all finite open covers $\mathcal{U}$. When $(Y, S)$ is the trivial (one-point) system, we call $h(T \mid S)$ the topological entropy of $(X, T)$, and denote it simply by $h(T)$.

In Section 1, we use the notation $h(X, T)$ to denote the topological entropy, but throughout the rest of the paper we use the notation $h(T)$ (noting that the dependence on $X$ is implicit, as it is the domain of the map $T)$. We remark that the above limit always exists and is independent of the choice of Følner sequence. Furthermore, if $(X, T)$ is a subshift and $\mathcal{U}$ is the clopen partition of $X$ according to the symbol at the identity element of $G$, then $\mathcal{U}$ is generating, and therefore $h(T \mid$ $S)=h(T, \mathcal{U} \mid S)$. Lastly, we note that $h(T \mid S) \leq h(T)$. 
It is well-known that for any countable amenable group $G$, factor maps cannot increase topological entropy, i.e. if $\varphi$ is a factor map from $(X, T)$ to $(Y, S)$, then $h(T) \geq h(S)$. This observation motivates the main question of this paper, namely whether a large set of entropies is achieved for intermediate systems 'between $X$ and $Y$.'

In the other direction, it is known that the decrease in topological entropy under a factor map is bounded above by the topological conditional entropy. Results of this type have been established by Bowen [1] and by Ledrappier and Walters [7] for $\mathbb{Z}$ actions. We require the following corollary of results from [15].

Theorem 2.16 ([15]). For any factor map $\pi: X \rightarrow Y$ between $G$ systems $(X, T)$ and $(Y, S)$,

$$
h(T) \leq h(S)+h(T \mid S) .
$$

2.4. Topological joinings. Suppose $(X, T)$ and $(Y, S)$ are $G$-systems. A topological joining of these systems is a $G$-system $(Z, R)$ such that $Z \subset X \times Y$, the action $R$ is defined by $R^{g}(x, y)=\left(T^{g}(x), S^{g}(y)\right)$, and the natural projections of $Z$ onto each its components gives factor maps onto $(X, T)$ and $(Y, S)$, respectively. For any such topological joining, we note that $h(R) \leq h(S)+h(T)$ (as $(Z, R)$ is a subsystem of the direct product system, which has entropy $h(S)+h(T))$.

Here we describe a specific construction of topological joinings that we use in some of our proofs. Suppose that $(X, T),(Y, S)$, and $(Z, R)$ are three $G$-systems, and $\pi: X \rightarrow Y$ and $\varphi: X \rightarrow Z$ are factor maps. Then we define $J(\pi, \varphi)$, the topological joining of $\pi$ and $\phi$, to be another $G$-system $(W, Q)$, defined as follows. The space $W$ is

$$
W=\{(\pi(x), \varphi(x)): x \in X\},
$$

and the action $Q$ is given by $Q^{g}(y, z)=\left(S^{g}(y), R^{g}(z)\right)$. Note that there are three factor maps associated to this construction:

- $\psi: X \rightarrow W$, where $\psi(x)=(\pi(x), \varphi(x))$,

- $\pi_{1}: W \rightarrow Y$, where $\pi_{1}(y, z)=y$, and

- $\pi_{2}: W \rightarrow Z$, where $\pi_{2}(y, z)=z$.

We state the following fact for future use.

Lemma 2.17. Suppose $\left(X_{0}, T_{0}\right),\left(X_{1}, T_{1}\right)$, and $(Y, S)$ are $G$-systems and $\pi: X_{0} \rightarrow X_{1}$ is a factor map. If $\left(W_{i}, R_{i}\right)$ is a topological joining of $\left(X_{i}, T_{i}\right)$ and $(Y, S)$ for $i=0,1$ and $\varphi: W_{0} \rightarrow W_{1}$ is the factor map defined by $\varphi(x, y)=(\pi(x), y)$, then $h\left(R_{0} \mid R_{1}\right) \leq h\left(T_{0} \mid T_{1}\right)$.

Proof. Let $\mathcal{U}$ be a finite open cover of $W_{0}$. Since the projection maps from $W_{0}$ onto $X_{0}$ and $Y$ are open maps, the projections of $\mathcal{U}$ onto 
these factors yield open covers $\mathcal{U}^{0}$ of $X_{0}$ and $\mathcal{U}^{Y}$ of $Y$. Also, for any $(x, y) \in W_{1}$, note that $\varphi^{-1}(x, y)=\pi^{-1}(x) \times\{y\}$. Hence, for any $n$, we have $N\left(\mathcal{U}_{F_{n}} \mid \varphi^{-1}(x, y)\right) \leq N\left(\mathcal{U}_{F_{n}}^{0} \mid \pi^{-1}(x)\right) \cdot N\left(\mathcal{U}_{F_{n}}^{Y} \mid\{y\}\right)=N\left(\mathcal{U}_{F_{n}}^{0} \mid\right.$ $\left.\pi^{-1}(x)\right)$. Then the desired inequality follows from the definition of topological conditional entropy.

2.5. Zero-dimensional systems. A $G$-system $(X, T)$ is said to be zero-dimensional if $X$ has a (topological) basis consisting of clopen sets. Suppose that $X$ is a zero-dimensional $G$ system. Then for any $\epsilon>0$, the space $X$ has a finite clopen partition $\mathcal{P}$ with $\operatorname{diam}(\mathcal{P})<\epsilon$. For $x \in X$, let $\mathcal{P}(x)$ be the element of $\mathcal{P}$ containing $x$. Then define a map $\pi_{\mathcal{P}}: X \rightarrow \mathcal{P}^{G}$ by the rule $\pi_{\mathcal{P}}(x)(g)=\mathcal{P}\left(T^{g}(x)\right)$, and note that $\pi$ is a factor map from $X$ onto $X_{\mathcal{P}}:=\pi_{\mathcal{P}}(X)$. In fact, by choosing $\epsilon>0$ sufficiently small, one may ensure that the entropy of the subshift $X_{\mathcal{P}}$ is arbitrarily close to the entropy of $(X, T)$.

\subsection{Inverse limits.}

Definition 2.18. Suppose $\left\{\left(Z_{n}, R_{n}\right)\right\}_{n=0}^{\infty}$ is a sequence of $G$-systems, and for each $n \geq 1$, we have a factor map $\pi_{n}: Z_{n} \rightarrow Z_{n-1}$. The inverse limit system $(Z, R)=\underset{\lim }{\longleftarrow}\left(Z_{n}, R_{n}\right)$ is defined as follows:

$$
Z=\left\{\left(z_{0}, z_{1}, z_{2}, \ldots\right) \in \prod_{n=0}^{\infty} Z_{n}: \forall n \geq 1, \pi_{n}\left(z_{n}\right)=z_{n-1}\right\},
$$

and $R^{g}\left(z_{0}, z_{1}, z_{2}, \ldots\right)=\left(R_{0}^{g}\left(z_{0}\right), R_{1}^{g}\left(z_{1}\right), R_{2}^{g}\left(z_{2}\right), \ldots\right)$.

We remark that since each $\left(Z_{n}, R_{n}\right)$ is a $G$-system, so is the inverse limit system $(Z, R)$. Furthermore, if each $Z_{n}$ is zero-dimensional, then $Z$ is zero-dimensional as well. We require the following lemma about entropy of inverse limits; for a proof in the case $G=\mathbb{Z}$, see $[9$, Lemma 4.9]. The straight-forward adaptation of this proof for general countable amenable groups is left to the reader.

Lemma 2.19. For any inverse limit system $(Z, R)=\underset{\swarrow}{\lim }\left(Z_{n}, R_{n}\right)$, we have

$$
h(R)=\lim _{n} h\left(R_{n}\right) .
$$

Finally, we will need the following simple lemma, which shows that factor maps can be carried to the inverse limit; the proof is standard and left to the reader.

Lemma 2.20. Suppose that $X$ is a $G$-system with a surjective factor map $\varphi_{0}: X \rightarrow Z_{0}$ and that for each $n \geq 1$, there exist surjective factor maps $\varphi_{n}: X \rightarrow Z_{n}$ and $\pi_{n}: Z_{n} \rightarrow Z_{n-1}$ such that $\varphi_{n-1}=\pi_{n} \circ \varphi_{n}$. 
Define the map $\varphi: X \rightarrow Z$ by the rule $\varphi(x)=\left(\varphi_{0}(x), \varphi_{1}(x), \ldots\right)$. Then $\varphi$ is a surjective factor map.

\subsection{Periodic patterns.}

Definition 2.21. For any finite alphabet $\mathcal{A}$, countable amenable $G$, $S, T \subset G$ with $S$ finite, $k \in \mathbb{N}$, and $w \in \mathcal{A}^{T}$, we say that $w$ has $k$ periods from $S$ if there exists a collection $\left\{s_{1}, \ldots, s_{k}\right\}$ of $k$ distinct elements of $S$ such that $w_{g}=w_{g s_{i}}$ whenever $g$ and $g s_{i}$ are both in $T$. In this case, we may refer to $\left\{s_{1}, \ldots, s_{k}\right\}$ as a period set for $w$.

Lemma 2.22. Let $k \in \mathbb{N}$, and let $S \subset G$ be a finite set with $|S| \geq k$. Let $T \subset G$ be any set such that

$$
k \log _{|\mathcal{A}|}|S|<\frac{|T|}{2 k}
$$

and for each $s \in S$,

$$
|T \triangle T s|<\frac{|T|}{2 k^{2}}
$$

Then

$$
\mid\left.\left\{w \in \mathcal{A}^{T}: w \text { has } k \text { periods from } S\right\}|\leq| \mathcal{A}\right|^{2|T| / k} .
$$

Proof. Let $k \in \mathbb{N}$, and let $S \subset G$ be a finite set with $|S| \geq k$. Let $T$ be as above. To simplify the notation in this proof, let $\delta>0$ be such that $\delta<k^{-1} / 2$ and such that

$$
k \log _{|\mathcal{A}|}|S|<\delta|T|
$$

and for each $s \in S$,

$$
|T \triangle T s|<\frac{\delta|T|}{k} .
$$

Now let $P \subset S$ such that $|P|=k$. First, define a finite undirected graph with vertex set $V=T$ and edge set $E \subset T \times T$, where $(g, h) \in E$ if there exists $p \in P$ such that $h=g p$ or $g=h p$. Let $C \subset T$ be the set of vertices corresponding to an arbitrary connected component of $(V, E)$ such that $|C|<k$ (which might not exist). Let $g \in C$. If $p \in P$ and $h=g p^{-1} \in T$, then $g=h p$, which means $(g, h) \in E$, and then $g p^{-1}=h \in C$. Since $|C|<k=\left|g P^{-1}\right|$, there exists $p \in P$ such that $g p^{-1} \notin T$, i.e., $g \in T \backslash T p$. We now conclude that for any connected component $C$ of $(V, E)$ with $|C|<k$, we have

$$
C \subset \bigcup_{p \in P}(T \backslash T p)
$$


For $g \in T$, let $C(g)$ denote the connected component of $(V, E)$ containing $g$. Then the above containment and our second hypothesis on $T$ combine to give

$$
|\{g \in T:|C(g)|<k\}| \leq \sum_{p \in P}|T \backslash T p|<\frac{\delta|T|}{k}|P|=\delta|T| .
$$

Let $N_{\ell}$ be the number of connected components of $(V, E)$ with cardinality $\ell$. We have

$$
\begin{aligned}
|T| & \geq|\{g \in T:|C(g)| \geq k\}| \\
& =\sum_{\ell=k}^{|T|} N_{\ell} \cdot \ell \\
& \geq k \sum_{\ell=k} N_{\ell},
\end{aligned}
$$

and therefore the number of connected components of cardinality at least $k$ is bounded above by $|T| / k$.

Now we turn to counting patterns in $\mathcal{A}^{T}$ with period set $P$. Notice that if $w \in \mathcal{A}^{T}$ has $P$ as a period set, then $w$ must be constant on any connected component of the graph $(V, E)$. Then the estimates in the previous paragraph yield that

$\mid\left.\left\{w \in \mathcal{A}^{T}: P\right.$ is a period set for $\left.w\right\}|\leq| \mathcal{A}\right|^{|T| / k} \cdot|\mathcal{A}|^{\delta|T|}=|\mathcal{A}|^{\left(k^{-1}+\delta\right)|T|}$.

Finally, let us estimate the number of patterns in $\mathcal{A}^{T}$ with $k$ periods from $S$. Using the previous estimates and our first hypothesis on $T$, we have

$$
\begin{aligned}
\mid\left\{w \in \mathcal{A}^{T}:\right. & w \text { has } k \text { periods from } S\} \mid \\
& \leq \sum_{\substack{P \subset S \\
|P|=k}} \mid\left\{w \in \mathcal{A}^{T}: P \text { is a period set for } w\right\} \mid \\
& \leq|\mathcal{A}|^{\left(k^{-1}+\delta\right)|T|} \cdot|\{P \subset S:|P|=k\}| \\
& \leq|\mathcal{A}|^{\left(k^{-1}+\delta\right)|T|} \cdot|S|^{k} \\
& \leq|\mathcal{A}|^{\left(k^{-1}+\delta\right)|T|} \cdot|\mathcal{A}|^{\delta|T|} \\
& =|\mathcal{A}|^{\left(k^{-1}+2 \delta\right)|T|} .
\end{aligned}
$$

Since $\delta<k^{-1} / 2$, the proof of the lemma is complete. 
2.8. Other preliminaries. We denote the usual binary entropy function by $H:[0,1] \rightarrow \mathbb{R}$, where $H(x)=-x \log (x)-(1-x) \log (1-x)$, with the convention that $0 \cdot \log 0=0$. The following fact is elementary and presented without proof.

Lemma 2.23. For any $n$ and $\alpha<1 / 2$, we have

$$
\sum_{k=0}^{\lfloor\alpha n\rfloor}\left(\begin{array}{l}
n \\
k
\end{array}\right) \leq 2^{H(\alpha) n} .
$$

\section{Marker Lemma}

Here we will prove a Marker Lemma for countable groups which generalizes the classical Krieger Marker Lemma ([2], 6]). First, we require a definition.

Definition 3.1. Let $\mathcal{F}$ be a finite collection of sets. For $k \in \mathbb{N}$, we say that $\mathcal{F}$ is $k$-fold disjoint if for all collections $\left\{F_{1}, \ldots, F_{k}\right\}$ of $k$ distinct elements of $\mathcal{F}$, we have

$$
\bigcap_{\ell=1}^{k} F_{\ell}=\varnothing
$$

Now we state our general Marker Lemma. Note that it does not require amenability of the group $G$.

Lemma 3.2. Let $G$ be a countable group, and let $X$ be a G-subshift. Let $S \subset G$ and $T \subset G$ be finite, and let $1 \leq k \leq|S|$. Then there exists a clopen set $F \subset X$ such that

(1) the collection $\left\{\sigma^{s}(F): s \in S\right\}$ is $(k+1)$-fold disjoint, and

(2) if

$$
x \notin \bigcup_{s \in S^{-1} S} \sigma^{s}(F),
$$

then $x(T)$ has $k$ periods from $S^{-1} S$.

The classical Krieger Marker Lemma corresponds to the case $G=\mathbb{Z}$, $k=1, S=[0, n]$, and $T=[-n, n]$ for some $n \in \mathbb{N}$. Our use for arbitrary $k$ can be thought of as allowing shifts of the marker set to have weaker disjointness properties in exchange for stronger periodicity properties away from it.

Proof. Let $\mathcal{N}=\left\{w_{1}, \ldots, w_{r}\right\}$ be an enumeration of the patterns $w$ in $\mathcal{A}^{T}$ such that $w$ does not have $k$ periods from $S^{-1} S$. Inductively define 
the following sets. Let $A_{1}=\left[w_{1}\right]$. For $i=1, \ldots, r-1$, let

$$
A_{i+1}=\left[w_{i+1}\right] \backslash\left(\bigcup_{j=1}^{i} \bigcup_{s \in S^{-1} S} \sigma^{s}\left(A_{j}\right)\right) \text {. }
$$

Let $F=\bigcup_{i=1}^{r} A_{i}$. Note that $F$ is clopen.

To establish (1), suppose for contradiction that there exists $P=$ $\left\{p_{1}, \ldots, p_{k+1}\right\} \subset S$ with $|P|=k+1$ and there exists $x \in X$ with

$$
x \in \bigcap_{p \in P} \sigma^{p}(F) .
$$

Then for each $p \in P$, we have that $\sigma^{p^{-1}}(x) \in F=\bigcup_{i} A_{i}$. For each $p \in$ $P$, choose $i(p) \in\{1, \ldots, r\}$ such that $\sigma^{p^{-1}}(x) \in A_{i(p)}$. We claim that there exists $w \in \mathcal{N}$ such that $\sigma^{p^{-1}}(x) \in[w]$ for all $p \in P$. To see this, suppose for contradiction that there exist $p, q \in P$ such that $i(p) \neq i(q)$. Assume without loss of generality that $i(p)<i(q)$ (otherwise reverse the roles of $p$ and $q$ ). Then

$$
\sigma^{q^{-1}}(x) \in A_{i(q)} \cap\left(\sigma^{q^{-1} p}\left(A_{i(p)}\right)\right),
$$

which gives a contradiction, since $q^{-1} p \in S^{-1} S$ and $A_{i(q)}$ is defined to be disjoint from $\sigma^{s}\left(A_{i(p)}\right)$ for all $s \in S^{-1} S$. Hence, there exists $w \in \mathcal{N}$ such that $\sigma^{p^{-1}}(x) \in[w]$ for all $p \in P$. Therefore

$$
\sigma^{p_{1}^{-1}}(x) \in[w] \cap \sigma^{p_{1}^{-1} p_{2}}[w] \cap \cdots \cap \sigma^{p_{1}^{-1} p_{k+1}}[w] .
$$

Since $|P|=k+1$, the non-emptiness of the intersection in the previous display gives that the set $\left\{p_{1}^{-1} p_{2}, \ldots, p_{1}^{-1} p_{k+1}\right\}$ is a set of $k$ periods from $S^{-1} S$ for $w$. However, this contradicts the fact that $w \in \mathcal{N}$. Thus we have established (1).

Now let $x \in X$ such that $x(T)$ does not have $k$ periods from $S^{-1} S$. Then $x(T)=w_{i}$ for some $i=1, \ldots, r$. If $i=1$, then $x \in F$. If $i>1$, then either $x \in A_{i} \subset F$ or else there exists $j<i$ and $s \in S^{-1} S$ such that $x \in \sigma^{s}\left(A_{j}\right)$. In all cases, we obtain that

$$
x \in \bigcup_{s \in S^{-1} S} \sigma^{s}(F) .
$$

Taking the contrapositive, we conclude that if

$$
x \notin \bigcup_{s \in S^{-1} S} \sigma^{s}(F),
$$

then $x(T)$ has $k$ periods from $S^{-1} S$. This establishes (2) and finishes the proof. 


\section{Density}

In this section, we define some basic notions of (upper) density for subsets of countable amenable $G$ (in terms of our previously chosen Følner sequence $F_{n}$ ). This will be used to quantify a way in which visits to the marker set from Lemma 3.2 are rare when $k$ is taken much smaller than $|S|$.

Definition 4.1. Let $X$ be a $G$-subshift, and let $F \subset X$. For a finite set $E \subset G$ and $x \in X$, let

$$
N_{E}(x, F)=\left|\left\{g \in E: \sigma^{g}(x) \in F\right\}\right| .
$$

Then let

$$
D_{n}(F)=\sup _{x \in X} \frac{N_{F_{n}}(x, F)}{\left|F_{n}\right|}
$$

and $\bar{D}(F)=\lim \sup _{n} D_{n}(F)$.

Definition 4.2. Let $X$ be a $G$-subshift. Let $S \subset G$ be finite, and let $k \in \mathbb{N}$. We say that $F \subset X$ is $(S, k)$-disjoint if $\left\{\sigma^{s}(F): s \in S\right\}$ is $k$-fold disjoint.

Lemma 4.3. Let $X$ be a $G$-subshift. Let $\left\{S_{1}, \ldots, S_{N}\right\}$ be a collection that $\delta$-quasitiles $G$ with $m=\min _{i}\left|S_{i}\right|$ and $S=\bigcup_{i} S_{i}=S_{N}$, and let $k \geq 1$. If $F \subset X$ is $\left(S^{-1}, k\right)$-disjoint, then

$$
\bar{D}(F) \leq \frac{k(1+\delta)}{(1-\delta) m}+\delta .
$$

Proof. By Lemma 2.5, for all large enough $n$, there exists $C=C(S, n, \delta)$ such that $\{S c: c \in C\}(1-\delta)$-covers $F_{n}$ and

$$
|C| \leq \frac{(1+\delta)\left|F_{n}\right|}{(1-\delta) m}
$$

Also, since $F$ is $\left(S^{-1}, k\right)$-disjoint, for any $x \in X$, we have

$$
\begin{aligned}
N_{S}(x, F) & =\left|\left\{g \in S: \sigma^{g}(x) \in F\right\}\right| \\
& =\left|\left\{g \in S: x \in \sigma^{g^{-1}}(F)\right\}\right| \\
& \leq k
\end{aligned}
$$


Then for any $x \in X$, the previous two displays and the fact that $\{S c: c \in C\}(1-\delta)$-covers $F_{n}$ gives that

$$
\begin{aligned}
N_{F_{n}}(x, F) & \leq N_{S C}(x, F)+N_{F_{n} \backslash S C}(x, F) \\
& \leq \sum_{c \in C} N_{S c}(x, F)+\left|F_{n} \backslash S C\right| \\
& \leq \sum_{c \in C} N_{S}\left(\sigma^{c}(x), F\right)+\delta\left|F_{n}\right| \\
& \leq k|C|+\delta\left|F_{n}\right| \\
& \leq \frac{k(1+\delta)\left|F_{n}\right|}{(1-\delta) m}+\delta\left|F_{n}\right| .
\end{aligned}
$$

After dividing by $\left|F_{n}\right|$, taking the supremum over $x \in X$, and letting $n$ tend to infinity, we obtain the desired estimate.

Now we show that if a factor map only changes a small percentage of symbols, then the topological conditional entropy is small, which implies by Theorem 2.16 that the entropy drop over the factor is also small.

Definition 4.4. Let $\pi: X \rightarrow Y$ be a factor map between $G$-subshifts. For a finite set $E \subset G, y \in Y$, and $x \in \pi^{-1}(y)$, we define

$$
N_{E}(x, y)=\left|\left\{g \in E: x_{g} \neq y_{g}\right\}\right| \text {. }
$$

Then define

$$
D_{n}(y)=\sup _{x \in \pi^{-1}(y)} \frac{N_{F_{n}}(x, y)}{\left|F_{n}\right|},
$$

and $\bar{D}(\pi)=\lim \sup _{n} \sup _{y \in Y} D_{n}(y)$.

Lemma 4.5. Let $\pi: X \rightarrow Y$ be a factor map between $G$-subshifts $(X, T)$ and $(Y, S)$ on alphabet $\mathcal{A}$. Suppose that $\bar{D}(\pi)<1 / 2$. Then $h(T \mid S) \leq H(\bar{D}(\pi))+\bar{D}(\pi) \log |\mathcal{A}|$.

Proof. Let $\gamma=\bar{D}(\pi)$, and let $\epsilon>0$ be such that $\gamma+\epsilon<1 / 2$. Choose $n$ large enough so that for all $y \in Y$ and $x \in \pi^{-1}(y)$, we have

$$
\frac{N_{F_{n}}(x, y)}{\left|F_{n}\right|}<\gamma+\epsilon
$$

Fix any $y \in Y$. For any $n$, define $\mathcal{L}_{F_{n}}\left(\pi^{-1}(y)\right)=\left\{x\left(F_{n}\right): x \in\right.$ $\left.\pi^{-1}(y)\right\}$. For any $x \in \pi^{-1}(y)$, define $K_{n, x}=\left\{g \in F_{n}: x_{g} \neq y_{g}\right\}$, and note that

$$
\left|K_{n, x}\right|=N_{F_{n}}(x, y) \leq(\gamma+\epsilon)\left|F_{n}\right|
$$


Since $y$ is fixed, we see that $x\left(F_{n}\right)$ may be determined by $K_{n, x}$ (off of which $x$ is equal to $y$ ) and $x\left(K_{n, x}\right)$, and therefore

$$
\left|\mathcal{L}_{F_{n}}\left(\pi^{-1}(y)\right)\right| \leq 2^{H(\gamma+\epsilon)\left|F_{n}\right|} \cdot|\mathcal{A}|^{(\gamma+\epsilon)\left|F_{n}\right|},
$$

where we have used Lemma 2.23 .

Taking logarithms, dividing by $\left|F_{n}\right|$, and letting $n$ tend to infinity gives

$$
h(T \mid S) \leq H(\gamma+\epsilon)+(\gamma+\epsilon) \log |\mathcal{A}|
$$

Since $\epsilon$ may be taken arbitrarily small, we obtain the desired result.

The following corollary is immediate via Theorem 2.16 .

Corollary 4.6. Under the assumptions of Lemma 4.5. $h(T)-h(S) \leq$ $H(\bar{D}(\pi))+\bar{D}(\pi) \log |\mathcal{A}|$.

\section{Proof of Theorem 1.1}

In this section we present the proof of Theorem 1.1. The main ingredient in the proof is Proposition 5.1. We present this proposition first and then apply it in the proof of the main theorem below.

Proposition 5.1. Let $(X, R)$ be a subshift. Then for any $\epsilon \in(0,1 / 2)$, there exists a finite sequence of subshifts $\left(X_{0}, R_{0}\right), \ldots,\left(X_{M+1}, R_{M+1}\right)$ and factor maps $\varphi_{m+1}: X_{m} \rightarrow X_{m+1}$ for $0 \leq m \leq M$ such that $\left(X_{0}, R_{0}\right)=(X, R),\left(X_{M+1}, R_{M+1}\right)$ is the trivial (one-point) system, and $h\left(R_{m} \mid R_{m+1}\right)<\epsilon$ for all $0 \leq m \leq M$.

Proof. Let $(X, R)$ be a $G$-subshift with alphabet $\mathcal{A}$. We assume that $|\mathcal{A}| \geq 2$, since when $|\mathcal{A}|=1$, we have $h(R)=0$ and the proposition holds trivially.

Let $\epsilon \in(0,1 / 2)$. Choose $k \geq 1$ such that $4 \log (|\mathcal{A}|) / k<\epsilon$. Choose $\delta \in(0,1)$ and $m_{0} \geq 1$ such that

$$
H\left(\frac{(k+1)(1+\delta)}{(1-\delta) m_{0}}+\delta\right)+\left(\frac{(k+1)(1+\delta)}{(1-\delta) m_{0}}+\delta\right) \log |\mathcal{A}|<\epsilon / 2
$$

Choose a collection $\left\{S_{1}, \ldots, S_{M}\right\}$ that $\delta$-quasitiles $G$ such that $\min _{i}\left|S_{i}\right| \geq$ $m_{0}$ and $S=\bigcup_{i} S_{i}$ has cardinality at least $k$. Choose $\eta \in(0,1)$ such that

$$
\log (|\mathcal{A}|)\left(2 k^{-1}(1-\eta)^{-1}+\eta\right)<\epsilon / 2 .
$$

Choose a collection $\left\{T_{1}, \ldots, T_{N}\right\}$ that $\eta$-quasitiles $G$ such that $2 k^{2} \log _{|\mathcal{A}|}\left|S S^{-1}\right| \leq\left|T_{1}\right|=\min _{i}\left|T_{i}\right|$ and for all $s \in S S^{-1}$ and $i=$ $1, \ldots, N$, we have

$$
\left|T_{i} \triangle T_{i} s\right|<\frac{\left|T_{i}\right|}{2 k^{2}}
$$


Let $T=\bigcup_{i} T_{i}$.

Now apply the Marker Lemma (Lemma 3.2) with parameters $k, S^{-1}$, and $T$. We get a clopen set $F \subset X$ such that $F$ is $\left(S^{-1}, k+1\right)$-disjoint and if $x \in X$ satisfies

$$
x \notin \bigcup_{s \in S S^{-1}} \sigma^{s}(F),
$$

then $x(T)$ has $k$ periods in $S S^{-1}$. By Lemma 4.3, we obtain that

$$
\bar{D}(F) \leq \frac{(k+1)(1+\delta)}{(1-\delta) m_{0}}+\delta
$$

Before defining our factor maps, we require a few more definitions. Let $G=\left\{g_{k}\right\}_{k=1}^{\infty}$ be an enumeration of $G$, with the convention that $g_{1}=e$. Let $G_{m}=\left\{g_{1}, \ldots, g_{m}\right\}$. We suppose that $a$ and $b$ are symbols that are not contained in $\mathcal{A}$, and we let $\mathcal{B}=\mathcal{A} \cup\{a, b\}$.

Now we define our factor maps. First, let $\varphi_{1}: X \rightarrow \mathcal{B}^{G}$ be defined by the rule

$$
\varphi_{1}(x)_{g}= \begin{cases}a, & \sigma^{g}(x) \in F \\ x_{g}, & \text { otherwise. }\end{cases}
$$

Since $F$ is clopen, $\varphi_{0}$ is a sliding block code. Let $X_{1}=\varphi_{1}(X)$. Inductively, suppose that $\varphi_{1}, \ldots, \varphi_{m}$ and $X_{1}, \ldots, X_{m}$ have been defined. Define $\varphi_{m+1}: X_{m} \rightarrow \mathcal{B}^{G}$ by the rule

$$
\varphi_{m+1}(x)_{g}= \begin{cases}b, & \text { if } x_{g} \neq a \text { and } x_{g_{m+1}^{-1} g}=a \\ x_{g}, & \text { otherwise. }\end{cases}
$$

It is clear that $\varphi_{m+1}$ is a sliding block code. Let $X_{m+1}=\varphi_{m+1}\left(X_{m}\right)$. This concludes our definition of the factor maps $\left\{\varphi_{m}\right\}_{m=0}^{\infty}$ and the subshifts $\left\{\left(X_{m}, R_{m}\right)\right\}_{m=0}^{\infty}$.

The remainder of the proof will be devoted to showing that the set of entropies of the subshifts $\left(X_{m}, R_{m}\right)$ is $\epsilon$-dense in the interval $[0, h(R)]$ by verifying that the topological conditional entropies $h\left(R_{m} \mid R_{m+1}\right)$ are smaller than $\epsilon$ and that the entropy $h\left(R_{m}\right)$ is 'eventually small,' i.e. $h\left(R_{m}\right)<\epsilon$ for sufficiently large $m$.

Both claims will be proved by appealing to properties of $F$ guaranteed by the Marker Lemma. The former will follow from the fact that visits to $F$ have small density, meaning that the changes made via each $\varphi_{m}$ have small density. The latter will follow from the fact that portions of points of $X$ which are not near visits to $F$ are highly periodic, and since letters at locations near visits to $F$ are changed to $a$ for large $m$, such $\left(X_{m}, R_{m}\right)$ will have small entropy by Lemma 2.22 ,

Now we will establish that each topological conditional entropy satisfies $h\left(R_{m} \mid R_{m+1}\right)<\epsilon$. 
Claim 5.2. $\bar{D}\left(\varphi_{1}\right) \leq \bar{D}(F)$.

(In fact, this inequality is an equality, but we will not need that fact.)

Proof. Let $\epsilon_{1}>0$. Choose $n$ large enough so that $D_{n}(F) \leq \bar{D}(F)+\epsilon_{1}$. Let $y \in X_{1}$ and $x \in \varphi_{1}^{-1}(y)$. Then

$$
\begin{aligned}
N_{F_{n}}(x, y) & =\left|\left\{g \in F_{n}: x_{g} \neq y_{g}\right\}\right| \\
& =\left|\left\{g \in F_{n}: \sigma^{g}(x) \in F\right\}\right| \\
& =N_{F_{n}}(x, F) \\
& \leq\left(\bar{D}(F)+\epsilon_{1}\right)\left|F_{n}\right|
\end{aligned}
$$

Dividing by $\left|F_{n}\right|$, taking supremum over $y \in Y$ and $x \in \varphi_{1}^{-1}(y)$, and letting $n$ tend to infinity yields

$$
\bar{D}\left(\varphi_{1}\right) \leq \bar{D}(F)+\epsilon_{1}
$$

Since $\epsilon_{1}$ may be taken arbitrarily small, we obtain that $\bar{D}\left(\varphi_{1}\right) \leq \bar{D}(F)$.

Note that $\bar{D}(F)<1 / 2$ (by (5.1) and (5.3)). Then by Corollary 4.6 and Claim 5.2, we see that

$$
\begin{aligned}
h\left(R \mid R_{1}\right) & \leq H\left(\bar{D}\left(\varphi_{1}\right)\right)+\bar{D}\left(\varphi_{1}\right) \log |\mathcal{A}| \\
& \leq H(\bar{D}(F))+\bar{D}(F) \log |\mathcal{A}| .
\end{aligned}
$$

Then by (5.1) and (5.3), we conclude that $h\left(R \mid R_{1}\right)<\epsilon$.

Claim 5.3. For all $m \geq 1$, we have $\bar{D}\left(\varphi_{m+1}\right) \leq \bar{D}(F)$.

Proof. Let $\epsilon_{1}>0$. Choose $n$ large enough so that $D_{n}(F) \leq \bar{D}(F)+\epsilon_{1}$ and $\left|\left(g_{m+1}^{-1} F_{n}\right) \backslash F_{n}\right| \leq \epsilon_{1}\left|F_{n}\right|$. Let $y \in X_{m+1}$ and $x \in \varphi_{m+1}^{-1}(y)$. Let $z \in X$ be such that $x=\varphi_{m} \circ \ldots \varphi_{1}(z)$. Then

$$
\begin{aligned}
N_{F_{n}}(x, y) & =\left|\left\{g \in F_{n}: x_{g} \neq y_{g}\right\}\right| \\
& \leq \mid\left\{g \in F_{n}: x_{g} \neq a \text { and } x_{g_{m+1}^{-1} g}=a \mid\right. \\
& \leq\left|\left\{g \in F_{n}: x_{g_{m+1}^{-1} g}=a\right\}\right| \\
& =N_{g_{m+1}^{-1} F_{n}}(z, F) \\
& \leq N_{F_{n}}(z, F)+N_{\left(g_{m+1}^{-1} F_{n}\right) \backslash F_{n}}(z, F) \\
& \leq\left(\bar{D}(F)+\epsilon_{1}\right)\left|F_{n}\right|+\left|\left(g_{m+1}^{-1} F_{n}\right) \backslash F_{n}\right| \\
& \leq\left(\bar{D}(F)+\epsilon_{1}\right)\left|F_{n}\right|+\epsilon_{1}\left|F_{n}\right| .
\end{aligned}
$$

Dividing by $\left|F_{n}\right|$, taking the supremum over $y \in X_{m+1}$ and $x \in \varphi_{m+1}^{-1}(y)$, and letting $n$ tend to infinity yields

$$
\bar{D}\left(\varphi_{m+1}\right) \leq \bar{D}(F)+2 \epsilon_{1} .
$$


Since $\epsilon_{1}$ may be taken arbitrarily small, we obtain that $\bar{D}\left(\varphi_{m+1}\right) \leq$ $\bar{D}(F)$.

By Corollary 4.6 and the previous claim, we see that

$$
\begin{aligned}
h\left(R_{m} \mid R_{m+1}\right) & \leq H\left(\bar{D}\left(\varphi_{m+1}\right)\right)+\bar{D}\left(\varphi_{m+1}\right) \log |\mathcal{A}| \\
& \leq H(\bar{D}(F))+\bar{D}(F) \log |\mathcal{A}| .
\end{aligned}
$$

Then by (5.1) and (5.3), we conclude that $h\left(R_{m} \mid R_{m+1}\right)<\epsilon$.

Now we proceed to show that $h\left(R_{m}\right)<\epsilon$ for sufficiently large $m$. Note that by our choice of the quasitiles $T_{1}, \ldots, T_{N}$, we may apply Lemma 2.22 with parameters $k, S S^{-1}$, and $T_{i}$, obtaining that for each $i$, we have

$$
\mid\left.\left\{v \in \mathcal{A}^{T_{i}}: v \text { has } k \text { periods from } S S^{-1}\right\}|\leq| \mathcal{A}\right|^{2\left|T_{i}\right| / k} .
$$

Lemma 5.4. For large enough $m$, we have $h\left(R_{m}\right)<\epsilon$.

Proof. Choose $m$ large enough that $T S S^{-1} \subset G_{m}$. Let $\delta_{1} \in(\bar{D}(F), 1 / 2)$ and $\delta_{2}>0$ be arbitrary. Choose $n$ large enough that $D_{n}(F) \leq \delta_{1}$ and

$$
\max \left(\frac{\left|F_{n} \triangle G_{m}^{-1} F_{n}\right|}{\left|F_{n}\right|}, \frac{\left|F_{n} \triangle T T^{-1} F_{n}\right|}{\left|F_{n}\right|}\right)<\delta_{2} .
$$

Since $\left\{T_{1}, \ldots, T_{N}\right\}$ is a set of $\eta$-quasitiles, there exists a collection $\left\{C_{1}, \ldots, C_{N}\right\}$ of center sets corresponding to $F_{n}$ with the additional property that if $c \in C_{i}$, then $T_{i} c \cap F_{n} \neq \varnothing$.

Let $w \in \mathcal{L}_{F_{n}}\left(X_{m}\right)$. Choose $y \in X_{m}$ such that $y\left(F_{n}\right)=w$, and choose $x \in X$ such that $y=\varphi_{m} \circ \ldots \varphi_{1}(x)$. Let $J_{w}=\left\{g \in G_{m}^{-1} F_{n}: y_{g}=a\right\}$. Note that for $g \in F_{n}$, we have that $w_{g}=a$ if and only if $g \in J_{w}$, and $w_{g}=b$ if and only if $g \in\left(G_{m} J_{w}\right) \backslash J_{w}$. Furthermore,

$$
\begin{aligned}
\left|J_{w}\right| & =\left|J_{w} \cap F_{n}\right|+\left|J_{w} \backslash F_{n}\right| \\
& \leq N_{F_{n}}(x, F)+\left|\left(G_{m}^{-1} F_{n}\right) \backslash F_{n}\right| \\
& \leq \delta_{1}\left|F_{n}\right|+\delta_{2}\left|F_{n}\right|,
\end{aligned}
$$

where we have used our choice of $n$ in the last estimate.

Now for each $i$, let $C_{i}^{w}$ be the set of $c \in C_{i}$ such that $\left(F_{n} \cap T_{i} c\right) \backslash$ $\left(G_{m} J_{w}\right) \neq \varnothing$. Note that $C_{i}^{w}$ is completely determined by $J_{w}$ (along with the already chosen $F_{n}, T_{i}$, and $\left.G_{m}\right)$.

Claim 5.5. If $c \in C_{i}^{w}$, then $x\left(T_{i} c\right)$ has $k$ periods from $S S^{-1}$.

Proof. To begin, suppose that $c \in C_{i}, g \in F_{n} \cap T_{i} c$, and

$$
\sigma^{c}(x) \in \bigcup_{s \in S S^{-1}} \sigma^{s}(F) \text {. }
$$


Then there exists $s \in S S^{-1}$ such that $\sigma^{s c}(x) \in F$. Hence $y_{s c}=a$. Let $g^{\prime}=s c$. Then $c=s^{-1} g^{\prime}$. Now let $t \in T_{i}$ be such that $t c=g$. Then $g^{\prime}=s t^{-1} g \in S S^{-1} T^{-1} F_{n} \subset G_{m}^{-1} F_{n}$, by our choice of $m$. Therefore $g^{\prime} \in J_{w}$, and then $g=t s^{-1} g^{\prime} \in T S S^{-1} J_{w} \subset G_{m} J_{w}$, again using our choice of $m$. We conclude that if $c \in C_{i}$ and

$$
\sigma^{c}(x) \in \bigcup_{s \in S S^{-1}} \sigma^{s}(F)
$$

then $F_{n} \cap T_{i} c \subset G_{m} J_{w}$. By the contrapositive, if $c \in C_{i}$ and $\left(F_{n} \cap T_{i} c\right) \backslash$ $\left(G_{m} J_{w}\right) \neq \varnothing$, then

$$
\sigma^{c}(x) \notin \bigcup_{s \in S S^{-1}} \sigma^{s}(F)
$$

which gives that $x(T c)$ has $k$ periods from $S S^{-1}$ by our choice of $F$. Thus, we have shown that if $c \in C_{i}^{w}$, then $x(T c)$ has $k$ periods from $S S^{-1}$, and therefore so does $x\left(T_{i} c\right)$ (since $T_{i} \subset T$ ).

For a finite set $E$, let $\mathcal{P}(E)$ denote the power set of $E$. Now consider the map $\phi: \mathcal{L}_{F_{n}}\left(X_{m}\right) \rightarrow \mathcal{P}\left(G_{m}^{-1} F_{n}\right)$ defined by $w \mapsto J_{w}$.

Claim 5.6. For each $J \subset \mathcal{P}\left(G_{m}^{-1} F_{n}\right)$, we have

$$
\left|\phi^{-1}(J)\right| \leq|\mathcal{A}|^{\eta\left|F_{n}\right|} \cdot|\mathcal{A}|^{(2 / k) \sum_{i}\left|T_{i}\right| \cdot\left|C_{i}\right|} .
$$

Proof. Let $J \in \mathcal{P}\left(G_{m}^{-1} F_{n}\right)$. Define $C_{i}^{\prime}=\left\{c \in C_{i}:\left(F_{n} \cap T_{i} c\right) \backslash\left(G_{m} J\right) \neq\right.$ $\varnothing\}$. Now let $w \in \phi^{-1}(J)$, i.e., $J_{w}=J$, and let $x \in X$ be such that $x\left(F_{n}\right)=w$. Note that since $J_{w}=J$, we also have $C_{i}^{w}=C_{i}^{\prime}$ for each $i$.

Let $g \in F_{n}$. For $g \in G_{m} J$, we have that $w_{g}=a$ whenever $g \in J$ and $w_{g}=b$ whenever $g \notin J$. Now suppose $g \in T_{i} c \backslash\left(G_{m} J\right)$ for some $c \in C_{i}$. Then $w_{g}=x_{g}$. Also, we have $c \in C_{i}^{\prime}$, and by Claim 5.5, $x\left(T_{i} c\right)$ has $k$ periods from $S S^{-1}$. Hence $w \in \phi^{-1}(J)$ is uniquely determined by a tuple of the form

$$
\left(\left(x\left(T_{1} c\right)\right)_{c \in C_{1}^{\prime}}, \ldots,\left(x\left(T_{N} c\right)\right)_{c \in C_{N}^{\prime}}, w\left(F_{n} \backslash\left(\bigcup_{i} T_{i} C_{i}\right)\right)\right)
$$

where each $x\left(T_{i} c\right)$ has $k$ periods from $S S^{-1}$. Thus, we have

$$
\begin{aligned}
&\left|\phi^{-1}(J)\right| \leq \prod_{i=1}^{N} \mid\left.\left\{v \in \mathcal{A}^{T_{i}}: v \text { has } k \text { periods from } S S^{-1}\right\}\right|^{\left|C_{i}^{\prime}\right|} \\
& \cdot \cdot|\mathcal{A}|^{\left|F_{n} \backslash\left(\bigcup_{i} T_{i} C_{i}\right)\right|} \\
& \leq \prod_{i=1}^{N} \mid\left.\left\{v \in \mathcal{A}^{T_{i}}: v \text { has } k \text { periods from } S S^{-1}\right\}\right|^{\left|C_{i}\right|} \\
& \cdot|\mathcal{A}|^{\left|F_{n} \backslash\left(\bigcup_{i} T_{i} C_{i}\right)\right|} .
\end{aligned}
$$


Then by Lemma 2.22 and the fact that $\left\{T_{1} C_{1}, \ldots, T_{N} C_{N}\right\}(1-\eta)$-covers $\left|F_{n}\right|$, we obtain the desired inequality

$$
\left|\phi^{-1}(J)\right| \leq|\mathcal{A}|^{\eta\left|F_{n}\right|} \cdot|\mathcal{A}|^{(2 / k) \sum_{i}\left|T_{i}\right| \cdot\left|C_{i}\right|} .
$$

Finally, using (5.5) in combination with Lemma 2.23 and Claim 5.6 yields the following estimate on the cardinality of $\left|\mathcal{L}_{F_{n}}\left(X_{m}\right)\right|$ :

$$
\left|\mathcal{L}_{F_{n}}\left(X_{m}\right)\right| \leq 2^{H\left(\delta_{1}+\delta_{2}\right)\left|F_{n}\right|}|\mathcal{A}|^{\eta\left|F_{n}\right|} \cdot|\mathcal{A}|^{(2 / k) \sum_{i}\left|T_{i}\right| \cdot\left|C_{i}\right|} .
$$

Using the $\eta$-disjointness of $T_{1}, \ldots, T_{N}$, we see that for each $i$, we have

$$
\begin{aligned}
\left|T_{i} C_{i}\right| & =\left|\bigcup_{c \in C_{i}} T_{i} c\right| \\
& \geq \sum_{c \in C_{i}}(1-\eta)\left|T_{i} c\right| \\
& \geq(1-\eta)\left|T_{i}\right| \cdot\left|C_{i}\right| .
\end{aligned}
$$

Recall that by our choice of centers, if $c \in C_{i}$, then $T_{i} c \cap F_{n} \neq \varnothing$. Let $g \in T_{i} c \cap F_{n}$. Then $g=t c$ for some $t \in T_{i}$, and so $c=t^{-1} g \in T^{-1} F_{n}$. Hence $T_{i} C_{i} \subset T T^{-1} F_{n}$. Combining the previous displayed formula with the quasi-invariance of $F_{n}$ with respect to $T T^{-1}$ and $\delta_{2}$ (by choice of $n)$, we obtain

$$
\begin{aligned}
\sum_{i}\left|T_{i}\right| \cdot\left|C_{i}\right| & \leq \frac{1}{1-\eta} \sum_{i}\left|T_{i} C_{i}\right| \\
& \leq \frac{1}{1-\eta}\left|\bigcup_{i} T_{i} C_{i}\right| \\
& \leq \frac{1}{1-\eta}\left|T T^{-1} F_{n}\right| \\
& \leq \frac{1+\delta_{2}}{1-\eta}\left|F_{n}\right| .
\end{aligned}
$$

Finally, putting together all of the above estimates, we get

$$
\begin{aligned}
\left|\mathcal{L}_{F_{n}}\left(X_{m}\right)\right| & \leq 2^{H\left(\delta_{1}+\delta_{2}\right)\left|F_{n}\right|}|\mathcal{A}|^{(2 / k) \sum_{i}\left|T_{i}\right| \cdot\left|C_{i}\right|}|\mathcal{A}|^{|\eta| F_{n} \mid} \\
& \leq 2^{H\left(\delta_{1}+\delta_{2}\right)\left|F_{n}\right|}|\mathcal{A}|^{(2 / k) \frac{1+\delta_{2}}{1-\eta}\left|F_{n}\right|}|\mathcal{A}|^{\left|F_{n}\right|}
\end{aligned}
$$

Taking logarithms, dividing by $\left|F_{n}\right|$, and letting $n$ tend to infinity, we get

$$
h\left(R_{m}\right) \leq H\left(\delta_{1}+\delta_{2}\right)+\left(\frac{2\left(1+\delta_{2}\right)}{k(1-\eta)}+\eta\right) \log |\mathcal{A}|
$$


Since $\delta_{2}>0$ was arbitrary, and since $\delta_{1} \in(\bar{D}(F), 1 / 2)$ was arbitrary, we see that

$$
h\left(R_{m}\right) \leq H(\bar{D}(F))+\left(\frac{2}{k(1-\eta)}+\eta\right) \log |\mathcal{A}| .
$$

By (5.1), (5.2), and (5.3), we conclude that $h\left(R_{m}\right)<\epsilon$. This finishes the proof of Lemma 5.4 .

Choosing $M$ sufficiently large, we have now established that $h\left(R_{M}\right)<$ $\epsilon$. Now let $\left(X_{M+1}, R_{M+1}\right)$ be the one-point system, which is trivially a factor of $\left(X_{M}, R_{M}\right)$ such that $h\left(R_{M} \mid R_{M+1}\right)=h\left(R_{M}\right)<\epsilon$. As a result, we have finished the proof of the proposition.

We are now prepared to prove Theorem 1.1

Proof of Theorem 1.1. Let $G$ and $(X, T)$ be as in the statement of the theorem. Let $\epsilon>0$. By Proposition 5.1, there exist subshifts $\left(X_{0}, T_{0}\right), \ldots,\left(X_{M+1}, T_{M+1}\right)$ and factor maps $\varphi_{m+1}: X_{m} \rightarrow X_{m+1}$ for $0 \leq m \leq M$ such that $\left(X_{0}, T_{0}\right)=(X, T),\left(X_{M+1}, T_{M+1}\right)$ is the onepoint system, and $h\left(T_{m} \mid T_{m+1}\right)<\epsilon$ for each $0 \leq m \leq M$. Note that each $\left(X_{m}, T_{m}\right)$ is a factor of $(X, T)$ (with factor map $\left.\varphi_{m} \circ \cdots \circ \varphi_{1}\right)$. Then by Theorem 2.16, we have that $h\left(T_{m}\right)-h\left(T_{m+1}\right) \leq h\left(T_{m} \mid T_{m+1}\right)<\epsilon$ for each $m=0, \ldots, M$. Also, since $\left(X_{M+1}, T_{M+1}\right)$ is the one-point system, we see that $h\left(T_{M}\right)<\epsilon$. Hence we have that $\left\{h\left(T_{m}\right): 0 \leq m \leq M\right\}$ is $\epsilon$-dense in $[0, h(T)]$. Since $\epsilon>0$ was arbitrary, we conclude that $\mathcal{H}_{\text {sub }}(X)$ is dense in $[0, h(T)]$, which finishes the proof of Theorem 1.1.

\section{Proof of Theorem 1.2}

Our first step is to prove an auxiliary result that will form the majority of the proofs of both Theorem 1.2 and Theorem 1.3. Informally, this result shows that the subshift factors $\left(X_{m}, T_{m}\right)$ from Proposition 5.1 still have $\epsilon$-dense entropies even after taking a topological joining with another $G$-system. (Recall from Section 2.4 that $J(\pi, \varphi)$ is our notation for the topological joining of two factor maps $\pi$ and $\varphi$ with common domain.)

Proposition 6.1. Suppose $(X, T)$ is a zero-dimensional $G$-system with finite entropy, $\varphi: X \rightarrow Z$ is a factor map onto a subshift $(Z, R)$, and $\pi: X \rightarrow Y$ is a factor map onto a $G$-system $(Y, S)$. Let $(W, Q)=$ $J(\varphi, \pi)$. Then for any $\epsilon>0$, there exist subshifts $\left(Z_{0}, R_{0}\right), \ldots,\left(Z_{M}, R_{M}\right)$ and factor maps $\psi_{m}: X \rightarrow Z_{m}$ for $0 \leq m \leq M+1$ such that if $\left(W_{m}, Q_{m}\right)=J\left(\psi_{m}, \pi\right)$, then $\left\{h\left(Q_{m}\right): 0 \leq m \leq M\right\}$ is $\epsilon$-dense in $[h(S), h(Q)]$. 
Proof. Assume the hypotheses of the proposition. Let $\epsilon>0$. By applying Proposition 5.1 to the subshift $(Z, R)$, we obtain that there exist subshifts $\left(Z_{0}, R_{0}\right), \ldots,\left(Z_{M+1}, R_{M+1}\right)$ and factor maps $\varphi_{m+1}: Z_{m} \rightarrow$ $Z_{m+1}$ for $0 \leq m \leq M$ such that $\left(Z_{0}, R_{0}\right)=(Z, R),\left(Z_{M+1}, R_{M+1}\right)$ is the one-point system, and $h\left(R_{m} \mid R_{m+1}\right)<\epsilon$ for $0 \leq m \leq M$. Define the factor maps $\psi_{m}: X \rightarrow Z_{m}$ by setting $\psi_{m}=\left(\varphi_{m} \circ \cdots \circ \varphi_{1}\right) \circ \varphi$ for each $1 \leq m \leq M$. Let $\left(W_{m}, Q_{m}\right)$ be the topological joining $J\left(\psi_{m}, \pi\right)$. It remains to show that $\left\{h\left(Q_{m}\right): 0 \leq m \leq M\right\}$ is $\epsilon$-dense in $[h(S), h(Q)]$.

First, note that $(W, Q)=\left(W_{0}, Q_{0}\right)$, and for $0 \leq m \leq M$, let $\varphi_{m+1} \otimes$ id $: W_{m} \rightarrow W_{m+1}$ be the factor map given by $\left(\varphi_{m+1} \otimes \mathrm{id}\right)(z, y)=$ $\left(\varphi_{m+1}(z), y\right)$. Then by Theorem 2.16] and Lemma 2.17, we have that for each $0 \leq m \leq M$, we have $h\left(Q_{m}\right)-h\left(Q_{m+1}\right) \leq h\left(Q_{m} \mid Q_{m+1}\right) \leq$ $h\left(R_{m} \mid R_{m+1}\right)<\epsilon$. Finally, since $\left(Z_{M+1}, R_{M+1}\right)$ is the one-point system and $h\left(R_{M} \mid R_{M+1}\right)<\epsilon$, we see that $h\left(R_{M}\right)=h\left(R_{M} \mid R_{M+1}\right)$ and then $h\left(Q_{m}\right) \leq h(S)+h\left(R_{M}\right)<h(S)+\epsilon$. Thus, we have established that $\left\{h\left(Q_{m}\right): 0 \leq m \leq M\right\}$ is $\epsilon$-dense in $[h(S), h(Q)]$.

Remark 6.2. We note for future reference that $\left(W_{m}, Q_{m}\right)$ are intermediate factors for $\pi$; indeed, they factor onto $Y$ via the projection $\pi_{2}$ to the second coordinate, and are factors of $(X, T)$, as they are topological joinings $J\left(\psi_{m}, \pi\right)$ of factors of $(X, T)$.

Proof of Theorem 1.2. Suppose $(X, T)$ and $(Y, S)$ are subshifts and $\pi: X \rightarrow Y$ is a factor map. For any $\epsilon>0$, if we define $(Z, R)=$ $(X, T)$ and let $\varphi: Z \rightarrow X$ be the identity map, then Proposition 6.1 yields intermediate factors $\left(W_{m}, Q_{m}\right)$ of $\pi$ for $0 \leq m \leq M$ whose entropies are $\epsilon$-dense in $[h(S), h(T)]$. Since each $\left(W_{m}, Q_{m}\right)$ is a subshift (as it is a topological joining of factors between subshifts) and $\epsilon>0$ was arbitrary, the proof is complete.

\section{Proof of Theorem 1.3}

Our proof proceeds by first using Proposition 6.1 to show that the entropies of zero-dimensional intermediate factors are dense in $[h(S), h(T)]$, and then using inverse limits to show that $\mathcal{H}_{0}^{\pi}(X, Y)=[h(S), h(T)]$.

Proof of Theorem 1.3. Suppose that $G,(X, T),(Y, S)$, and $\pi$ are as in the theorem. We begin by showing that the entropies of zero-dimensional intermediate factors are dense in $[h(S), h(T)]$.

Let $\epsilon>0$. Since $X$ is zero-dimensional, there exists a finite clopen partition $\mathcal{P}$ of $X$ such that $h\left(T_{\mathcal{P}}\right)>h(T)-\epsilon / 2$. By Proposition 6.1 (taking $(Z, R)$ to be $\left(X_{\mathcal{P}}, T_{\mathcal{P}}\right), \varphi=\pi_{\mathcal{P}}$, and parameter $\left.\epsilon / 2\right)$, there exist intermediate factors $\left(W_{m}, Q_{m}\right)$ of $\pi$ for $0 \leq m \leq M$ whose entropies 
are $\epsilon / 2$-dense in $\left[h(S), h\left(T_{\mathcal{P}}\right)\right]$, and therefore $\epsilon$-dense in $[h(S), h(T)]$. Since each $\left(W_{m}, Q_{m}\right)$ is zero-dimensional and $\epsilon>0$ was arbitrary, we conclude that $\mathcal{H}_{0}^{\pi}(X, Y)$ is dense in $[h(S), h(T)]$.

To complete the proof, we will construct zero-dimensional intermediate factors with arbitrary entropy $r \in[h(S), h(T)]$ as inverse limits of intermediate zero-dimensional factors using Lemma 2.20 .

The case $r=h(S)$ is trivial, and so we let $r \in(h(S), h(T)]$. Let $\left(Z_{0}, R_{0}\right)=(Y, S), \varphi_{0}=\pi$, and $\psi_{0}$ be the identity map on $Y$. By the density of $\mathcal{H}_{0}^{\pi}(X, Y)$ in $[h(S), h(T)]$, there exists a zero-dimensional system $\left(Z_{1}, R_{1}\right)$ and factor maps $\varphi_{1}: X \rightarrow Z_{1}$ and $\pi_{1}: Z_{1} \rightarrow Z_{0}$ such that $\pi=\pi_{1} \circ \psi_{1}$ and $h\left(R_{1}\right) \in(r-1, r)$. Now suppose we have defined $\left(Z_{n}, R_{n}\right), \varphi_{n}: X \rightarrow Z_{n}$, and $\pi_{n}: Z_{n} \rightarrow Z_{n-1}$. By the density of $\mathcal{H}_{0}^{\varphi_{n}}\left(X, Z_{n}\right)$ in $\left[h\left(R_{n}\right), h(T)\right]$, there exists a zero-dimensional system $\left(Z_{n+1}, R_{n+1}\right)$ and factor maps $\varphi_{n+1}: X \rightarrow Z_{n+1}$ and $\pi_{n+1}: Z_{n+1} \rightarrow Z_{n}$ such that $\pi_{n}=\pi_{n+1} \circ \varphi_{n+1}$ and $h\left(R_{n+1}\right) \in\left(r-\frac{1}{n+1}, r\right)$. Let $(Z, R)=$ $\lim \left(Z_{n}, R_{n}\right)$, and let $\varphi: X \rightarrow Z$ be the natural factor map (as in Lemma 2.20). Then $Z$ is an intermediate factor between $(X, T)$ and $(Y, S)$, and by Lemma 2.19 and our choice of $h\left(R_{n}\right)$ for each $n$, we have that $h(R)=\lim _{n} h\left(R_{n}\right)=r$, which concludes the proof of the theorem.

\section{REFERENCES}

[1] R. Bowen. Entropy for group endomorphisms and homogeneous spaces. Trans. Amer. Math. Soc., 153:401-414, 1971.

[2] M. Boyle. Lower entropy factors of sofic systems. Ergodic theory and dynamical systems, 3(04):541-557, 1983.

[3] J. Frisch and O. Tamuz. Symbolic dynamics on amenable groups: the entropy of generic shifts. Ergodic Theory Dynam. Systems, 37(4):1187-1210, 2017.

[4] S. Hong, U. Jung, and I.-J. Lee. Decompositions of factor codes and embeddings between shift spaces with unequal entropies. Ergodic Theory Dynam. Systems, 33(1):144-157, 2013.

[5] D. Kerr and H. Li. Ergodic theory. Springer Monographs in Mathematics. Springer, Cham, 2016. Independence and dichotomies.

[6] W. Krieger. On the subsystems of topological Markov chains. Ergodic Theory and Dynamical Systems, 2(02):195-202, 1982.

[7] F. Ledrappier and P. Walters. A relativised variational principle for continuous transformations. Journal of the London Mathematical Society, 2(3):568-576, 1977.

[8] D. Lind and B. Marcus. An introduction to symbolic dynamics and coding. Cambridge University Press, Cambridge, 1995.

[9] E. Lindenstrauss. Lowering topological entropy. J. Anal. Math., 67:231-267, 1995. 
[10] E. Lindenstrauss. Mean dimension, small entropy factors and an embedding theorem. Publications Mathématiques de l'Institut des Hautes Études Scientifiques, 89(1):227-262, 1999.

[11] E. Lindenstrauss and B. Weiss. Mean topological dimension. Israel Journal of Mathematics, 115(1):1-24, 2000.

[12] D. S. Ornstein and B. Weiss. Entropy and isomorphism theorems for actions of amenable groups. Journal d'Analyse Mathématique, 48(1):1-141, 1987.

[13] M. Shub and B. Weiss. Can one always lower topological entropy? Ergodic Theory Dynam. Systems, 11(3):535-546, 1991.

[14] M. Straszak. Topological dynamics of countable amenable group actions. $\mathrm{PhD}$ thesis, Jagiellonian University in Krakow, 2020.

[15] K. Yan. Conditional entropy and fiber entropy for amenable group actions. Journal of Differential Equations, 259(7):3004-3031, 2015.

Kevin MeGoff, Department of Mathematics and Statistics, University of North Carolina at Charlotte, 9201 University City Blvd., Charlotte, NC 28223

E-mail address: kmcgoff1@uncc.edu

$U R L:$ https://clas-math.uncc.edu/kevin-mcgoff/

Ronnie Pavlov, Department of Mathematics, University of Denver, 2390 S. York St., Denver, CO 80208

E-mail address: rpavlov@du.edu

$U R L:$ http://www.math.du.edu/ rpavlov/ 